\title{
Highly stretchable, compressible, adhesive hydrogels with double network
}

\author{
Cuiping Guo $0^{1,2,3} \cdot$ Zhiwen Zeng ${ }^{1,2,3} \cdot$ Shan $\mathrm{Yu}^{1,2,3} \cdot$ Xiaoyan Zhou $^{1,2,3} \cdot$ Qunfeng Liu ${ }^{1,2,3} \cdot$ Dating Pei ${ }^{1,2,3}$. \\ Daohuan Lu ${ }^{1,2,3} \cdot$ Zhijie Geng $^{1,2,3}$
}

Received: 9 June 2021 / Accepted: 20 September 2021 / Published online: 9 October 2021

(c) The Author(s) 2021

\begin{abstract}
In this work, a double network bovine serum albumin-polyacrylamide (BSA-PAM) adhesive hydrogel was fabricated, in which combination of physical interactions including hydrogen bonds and chain entanglements, and chemical covalent photocrosslinking. The BSA-PAM hydrogel exhibited excellent mechanical and adhesive properties. The composite hydrogel not only demonstrated excellent tensile properties (maximum force elongation 1552\% 2037\%), but also displayed extremely high fatigue resistance even when subjected to compress strains of up to $85 \%$. More importantly, the BSA-PAM hydrogel showed excellent adhesiveness to various substrates ( $90 \mathrm{kPa} 150 \mathrm{kPa}$ for glass and $9.74 \mathrm{kPa} 35.09 \mathrm{kPa}$ for pigskin). This work provided a facile way of fabricating tough, stretchable and adhesive BSA-PAM hydrogels.
\end{abstract}

Keywords Double network $\cdot$ Physical interactions $\cdot$ Chemical covalent photo-crosslinking $\cdot$ High strength $\cdot$ Protein adhesive

\section{Introduction}

Adhesive hydrogels were three-dimensional polymer networks that cross-linked either physically or chemically [1-3], which were used for tissue adhesion [4-8], hemostasis [9-12], and sealing against the leakage of air and body fluids during surgical procedures [13-15]. Various studies have been carried out to develop synthetic (e.g., cyanoacrylates) and protein [e.g., collagen,bovine serum albumin (BSA) and fibrin] adhesive products [16, 17]. However, synthetic and protein adhesives often exhibited limited bonding capacity and weak mechanical properties [18-21]. Therefore, adhesives owning high adhesive strength and mechanical properties were desired.

Cuiping Guo

guocui555@163.com

1 Institute of Medicine and Health, Guangdong Academy of Sciences, Guangzhou Road 1307, Guangzhou 510500, China

2 National Engineering Research Center for Healthcare Devices, Guangzhou Road 1307, Guangzhou 510500, China

3 Guangdong Key Laboratory of Medical Electronic Instruments and Polymer products, Guangzhou Road 1307, Guangzhou 510500, China
In the recent reports, Many efforts have been made to improve the adhesive and mechanical properties of hydrogels. Double network (DN) hydrogels have been fabricated [22-25]. The DN hydrogels were composed of a highly cross-linked network that endowed stiffness to the hydrogel, and a second loosely cross-linked one that endowed toughness to it [26]. For example, Huang and coworkers reported a series of tough and conductive DN hydrogels hybridized with 2,2,6,6-tetramethylpiperidine-1-oxy oxidized cellulose nanofiber (TOCN) and polypyrrole (Py), which exhibited high mechanical properties (the Young's modulus $\sim 1 \mathrm{MPa}$ ) and better tensile strength and strain $\left(\sigma_{\mathrm{Tb}}=451 \mathrm{kPa}\right.$ and $\varepsilon_{\mathrm{Tb}}=107 \%$ ) [27]. However, a facile way of preparing multifunctional double network adhesive hydrogels was still lacking.

BSA is a typical natural globular protein with high water solubility, biocompatibility and low production cost, which has been proposed as a biomaterial with a range of biomedical and industrial applications [28, 29]. These properties made BSA a good candidate as a tissue adhesive. Previously, BioGlue was a well-known BSA based tissue adhesive product [30], in which glutaraldehyde was used as a cross-linker for both inter- and intra- protein molecular. Although this adhesive showed good adhesive to tissues, its inconvenience, special tools usage might limit its use in clinic [31]. 
Besides, BioGlue had relatively low mechanical properties [30]. There were several hydrogels based on BSA reported for improving its mechanical properties [32-36], for example, Matsudomi, Tang et al. obtained self-supporting BSA based double-network hydrogels with high tensile strength, but it still challenged to obtain for complex process [37].

Acrylamide (AAM) is a cheap, easily available and highly hydrophilic monomer, which exhibited improved hydrolytic stability. Incorporation of AAM into adhesive can improve its mechanical properties. For example, Rodrigues et al. reported an experimental adhesive resin containing hydroxyethyl acrylamide used for dental adhesive resin, which showed that the addition of acrylamide not only increased the material's reactivity, but also improved the mechanical properties of dental adhesive resin [38, 39].

In this study, we have prepared double network adhesive hydrogels based on BSA and AAM by a facile one-pot synthesis method. All the reactants were mixed and stirred at R. T., and then AAM monomer was polymerized through UV curing method. The fabricated BSA-PAM hydrogel exhibited superior tensile properties and excellent adhesive properties. A series of studies were carried out to evaluate the mechanical, adhesive, swelling and rheological properties of BSA-PAM hydrogels.

\section{Materials}

BSA was purchased from Yuanye Bio-Technology Co., Ltd (Shanghai, China). $M_{w}$ was $68 \mathrm{kD}$, with a purity of $98 \%$. Acrylamide, $N, N^{\prime}$-methylene diacrylamide (BIS) and lithium phenyl-2, 4, 6-trimethylbenzoylphosphinate (LAP) were purchased from Yuanye Biochemical Co., Ltd. (Shanghai, China). All reagents were used as received. Fresh porcine skin was bought from local market. The Gelatin-coated glass slides $(25 \mathrm{~mm} \times 75 \mathrm{~mm})$ were purchased from Hito. BioTechnology Co., Ltd. (Guangzhou, SMAT agent, China).

\section{Synthesis of BSA-PAM hydrogels}

BSA $\left(5.0 \mathrm{~g}, 7.5 \times 10^{-5} \mathrm{~mol}\right)$, AAm $(2.5 \mathrm{~g} / 1.7 \mathrm{~g} / 1.3 \mathrm{~g}, 3.5 \times$ $\left.10^{-2} \mathrm{~mol} / 2.4 \times 10^{-2} \mathrm{~mol} / 1.8 \times 10^{-2} \mathrm{~mol}\right)$, LAP $(0.010 \mathrm{~g}, 3.4$ $\left.\times 10^{-5} \mathrm{~mol}\right)$, BIS $\left(0.0016 \mathrm{~g} / 0.0011 \mathrm{~g} / 0.0008 \mathrm{~g}, 1.0 \times 10^{-5}\right.$ $\mathrm{mol} / 0.71 \times 10^{-5} \mathrm{~mol} / 0.51 \times 10^{-5} \mathrm{~mol}$ ) were dissolved into distilled water $(10 \mathrm{~mL})$ in a reactor, and stirred about $30 \mathrm{~min}$ at $\mathrm{R}$. T. Then, the above solution was exposed under UV light $\left(365 \mathrm{~nm}, 30 \mathrm{~mW} \mathrm{~cm}^{-2}\right.$ ) for $3 \mathrm{~min}$. BSA-PAM-2, BSAPAM-3 and BSA-PAM-4 hydrogels were obtained.

\section{ATR-FTIR measurement}

All infrared spectra were recorded in the range of 400-3500 $\mathrm{cm}^{-1}$ using total reflectance fourier transform infrared
(ATR-FTIR) (Nicolet IS10). Each spectrum was acquired by accumulation of 32 scans with a resolution of $4 \mathrm{~cm}$. Background measurements were subtracted from the sample readings.

\section{SEM characterizations}

The structures of the BSA-PAM hydrogels were examined using a scanning electron microscope (SEM, TESCAN MIRA4). Before examination, the hydrogels were freezedried at $-60{ }^{\circ} \mathrm{C}$ for $48 \mathrm{~h}$. Then, the hydrogels were fractured by liquid nitrogen to expose their inner structures, and the cross-sections were observed.

\section{Lap shear test}

The lap shear strength property of BSA-PAM adhesive hydrogels was tested as previously described [40, 41]. One glass slide $(25 \mathrm{~mm} \times 75 \mathrm{~mm})$ and one fresh porcine skin $(25 \mathrm{~mm} \times 75 \mathrm{~mm})$ were used to hold each sample. The top portion of the glass slide $(20 \mathrm{~mm} \times 25 \mathrm{~mm})$ was coated with gelatin. The pre-polymer solution $(20 \mathrm{ml})$ was added to one of the gelatin-coated regions in glass slide, and then fresh porcine skin was carefully put on the solution. After that, the gel was photo-crosslinked by UV light $(365 \mathrm{~nm}$, $\left.30 \mathrm{~mW} \mathrm{~cm}{ }^{-2}\right)$. The sample was placed into a mechanical tester (KEJIAN, KJ-1065A, China) for shear testing by tensile loading with a strain rate of $1 \mathrm{~mm} / \mathrm{min}$. The lap shear strength was determined at the point of detaching.

\section{Tensile stress test}

Mechanical tensile stress-strain evaluation was conducted by a testing machine (KEJIAN, KJ-1065A, China) equipped with a $500 \mathrm{~N}$ tension sensor at room temperature. All gels $(\mathrm{n} \geq 4)$ were cast using polytetrafluoroethylene molds into dumbbell-shaped samples (1.5 mm thickness, $4.0 \mathrm{~mm}$ width at center). The tensile strength, elongation at rupture and Young's modulus were measured with an extension rate of $30 \mathrm{~mm} \mathrm{~s}^{-1}$. The Young's modulus was determined from the slope of the linear section stress-strain curve at 5\%-10\% strain.

\section{Cyclic compress test}

Cyclic loading-unloading tests were performed by a testing machine (KEJIAN, KJ-1065A, China). The hydrogels compressed to strains of $85 \%$ and then unloaded to zero force. The compress modulus was determined from the slope of the linear section stress-strain curve at 5\%-10\% strain. 


\section{Rheological measurements}

Rheological testing was carried out as previously described [42]. The testing was conducted on a Modular Compact Rheometer (Anton paar, MCR 302, Austria) at $25{ }^{\circ} \mathrm{C}$ using a $8 \mathrm{~mm}$ diameter flat plate with the gap set to $0.5 \mathrm{~mm}$. Excess sample was trimmed off the lower plate. The storage modulus $\left(\mathrm{G}^{\prime}\right)$ and loss modulus $\left(\mathrm{G}^{\prime \prime}\right)$ were measured and recored by performing a shear frequency-sweep test using a $1 \%$ strain and an angular frequency $(\omega)$ sweeping from 0.1 to $100 \mathrm{rad} \mathrm{s}^{-1}$.

\section{Swelling test}

For study of the swelling properties of the BSA-PAM hydrogel, the as-prepared cylindrical BSA-PAM hydrogels were dried, and weighed to find the initial dry weight $\left(\mathrm{W}_{\mathrm{i}}\right)$. The pre-weighed dry BSA-PAM hydrogels were immersed in $10 \mathrm{ml}$ distilled water or urea $(5 \mathrm{M})$ in distilled water separately. Samples were taken out from solution at each predetermined time points and weighed again $\left(\mathrm{W}_{\mathrm{t}}\right)$. Swelling ratio of the BSA-PAM hydrogel was determined by equation: Swelling ratio $=\left(\mathrm{W}_{\mathrm{t}}-\mathrm{W}_{\mathrm{i}}\right) / \mathrm{W}_{\mathrm{i}}$, where $\mathrm{W}_{\mathrm{i}}$ was the original dry weight and $\mathrm{W}_{\mathrm{t}}$ was the dry weight after incubation for a certain period.

\section{Statistical analysis}

All data were presented as means \pm standard deviations. All experiments were carried out with at least 3 parallel samples.

\section{Results and discussion}

\section{Preparation and characterization of BSA-PAM adhesive hydrogels}

In this work, the BSA-PAM adhesive hydrogel was fabricated by a simple one-pot synthesis method (Scheme 1). Briefly, all reactants of BSA, AAm, UV-initiator (LAP) and cross-linker (BIS) were dissolved into water in a reactor. The precursor solution was stirred at room temperature for $30 \mathrm{~min}$. Then, the double network BSA-PAM adhesive hydrogel was formed after UV irradiation $(365 \mathrm{~nm}, 30 \mathrm{~mW}$ $\mathrm{cm}^{-2}$ ). As shown in Fig. 1a, the first network was formed through physical interactions including hydrogen bonds and chain entanglements between BSA chains and AAM molecules. The second network was fabricated through chemical covalent crosslinking of AAM monomers by the cross-linker (BIS) under UV irradiation. The two networks induced a synergetic effect in enhancing the mechanical and adhesive properties of the BSA-PAM hydrogels. ATR-FTIR
Scheme 1 Fabrication process of the double-network BSAPAM hydrogel. (a) The BSAPAM hydrogels was formed. (b) Proposed molecular structure and interactions of the formed BSA-PAM hydrogel a

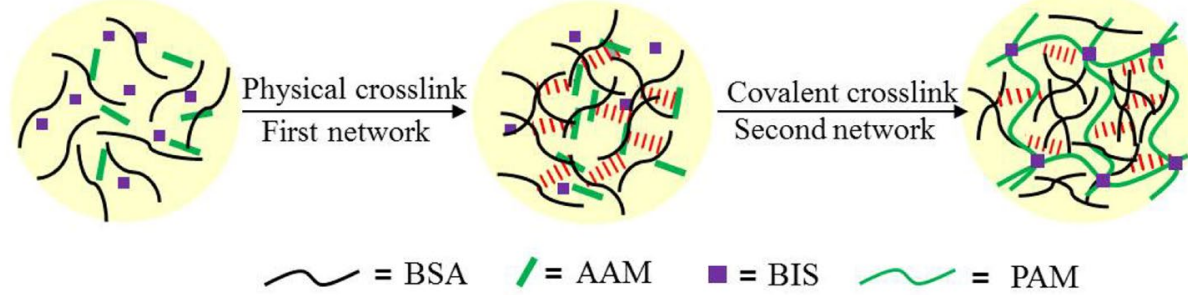

b

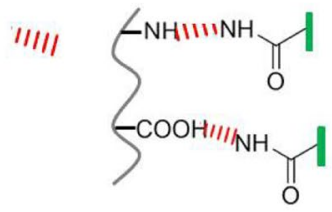

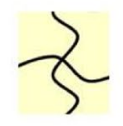

Hydrogen bonds

Chain entanglement
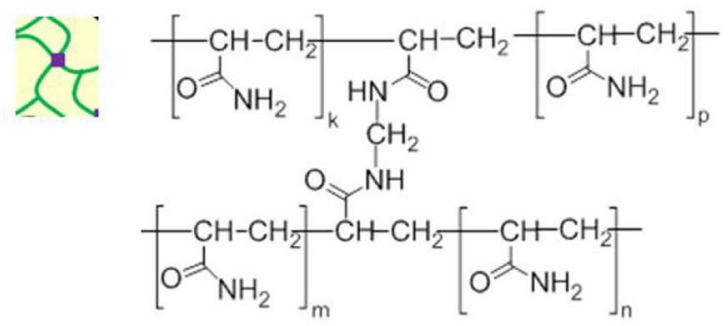

Covalent bonds 

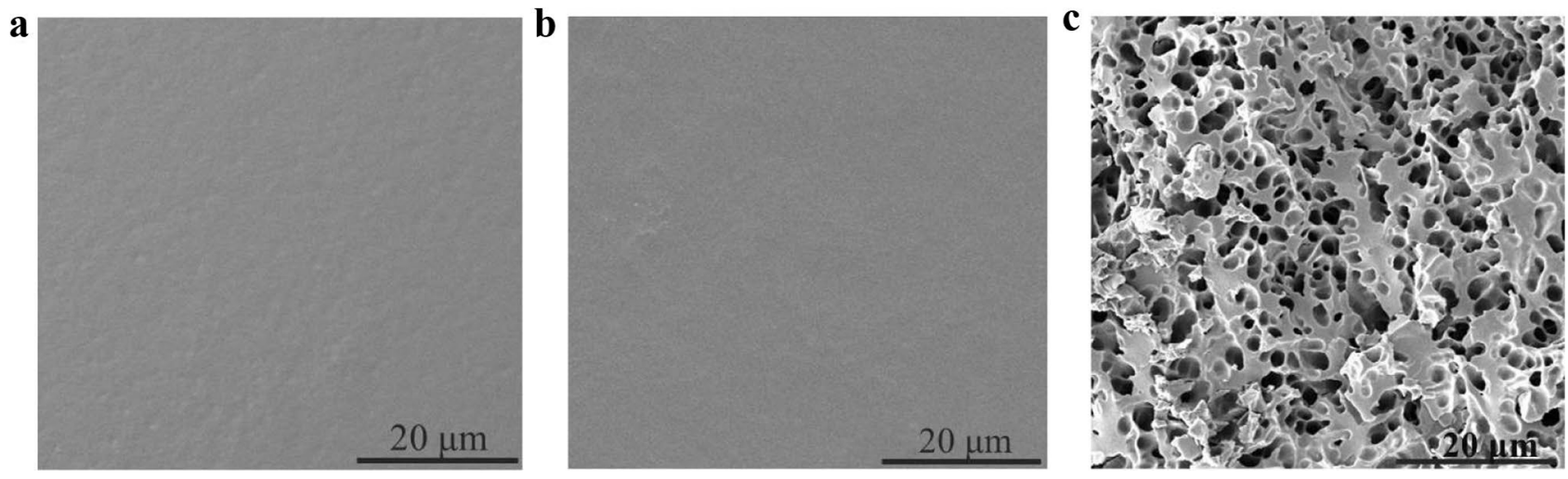

Fig. 1 Scanning electron microscopy (SEM) micrograph of the BSA-PAM hydrogels. (a) BSA-PAM-2. (b) BSA-PAM-3. (c) BSA-PAM-4

was utilized to analyze the structure and network interactions of the obtained hydrogels. As shown in Fig. S1, compared to pure BSA, there was a new peak of $1114 \mathrm{~cm}^{-1}$ for BSAPAM-2/BSA-PAM-3/BSA-PAM-4, which was assigned as the characteristic of C-N amide bond [36]. SEM micrographs showed that the pore size of the hydrogels gradually increased with weight ratio of BSA : AAM from 2:1 to 4:1 (Fig. 1).

The BSA-PAM adhesive hydrogel was prepared with different BSA: AAM weight ratios as listed in the supporting information (Fig. S2, Table S1). It was clear that the BSA-PAM hydrogel was well formed with weight ratio of BSA : AAM from $1: 1$ to $6: 1$, and we chose hydrogels formed with weight ratio of BSA : AAM from $2: 1$ to 4 $: 1$ as typical cases to study. Figure $1 \mathrm{~b}$ showed proposed molecular structure of the BSA-PAM hydrogel. Due to BSA protein molecules contained numerous functional groups such as $-\mathrm{NH}_{2}$ group, $-\mathrm{COOH}$ group, $-\mathrm{OH}$ group, etc., multiple hydrogen bonds were formed between BSA chains and AAM monomer molecules. Besides, chain entanglements were also formed between BSA chains. After UV irradiation, AAM monomers was polymerized to form PAM backbone, which was covalently cross-linked by the cross-linker (BIS). PAM backbone served as the primary network, and BSA chains inter-diffused and got physically entangled within the PAM network [43-45]. The abundant hydrogen bonds between the BSA chains and PAM network equipped the obtained hydrogels with high stretchability. Numerous functional groups such as $-\mathrm{NH}_{2}$ group, $-\mathrm{COOH}$ group and $-\mathrm{OH}$ group on BSA-PAM hydrogel equipped the obtained hydrogels with high adhesive ability to different substrates.

To verify the hypothesis that 'BSA-PAM hydrogels were largely cross-linked by hydrogen bonds between the BSA chains and PAM network, we used urea as hydrogen-bond-breaking agents to break hydrogen bonds existing in the BSA-PAM-2, BSA-PAM-3 and BSAPAM-4 hydrogel and studied their swelling kinetics. As shown in Fig. 7, all three hydrogels swelled obviously after soaking in water and urea (5 M) for 24 hours, and balanced after 48 hours. It was clear that all three hydrogels displayed much higher swelling ratio in urea than in water, due to the intermolecular hydrogen bonds had been destroyed, indicating that hydrogen bonding was an important driving force for the formation of BSA-PAM hydrogels.

\section{Mechanical properties of the BSA-PAM hydrogels}

The BSA-PAM hydrogels exhibited super stretchability and high toughness due to abundant physical interactions including hydrogen bonding and chain entanglements between BSA chains and PAM network. As shown in Fig. 2a, the BSA-PAM hydrogel could be easily stretched to 19 times and even twisted stretched (Fig. S3). To further quantitatively evaluate the mechanical behaviors of the prepared hydrogel, tensile tests were conducted. Figure 2b, c and d illustrate the typical tensile stress-strain curves of BSA-PAM hydrogels, corresponding maximum force elongation and Young's modulus.

It was obvious that the maximum force elongation could be stretched $1923.05 \pm 50.00 \%, 2037.85 \pm 54.00 \%$ and $1552.25 \pm 66.75 \%$ beyond its initial length for BSAPAM-2, BSA-PAM-3 and BSA-PAM-4 respectively (Fig. 2c, Table 1). Increasing weight ratio of BSA : AAM would decrease the Young's modulus from $2.56 \pm 0.12$ $\mathrm{kPa}, 2.24 \pm 0.36 \mathrm{kPa}$ to $1.20 \pm 0.24 \mathrm{kPa}$ for BSA-PAM-2, BSA-PAM-3 and BSA-PAM-4 respectively, due to the low cross-linking degree of the BSA-PAM hydrogel network (Fig. 2d). 
Fig. 2 (a) Diagram of tensile stress test: the original length (above), and the length after tensile stress (under). (b) Representative strain-stress curves for tensile stress test of BSAPAM-2, BSA-PAM-3 and BSAPAM-4. (c) The maximum force elongation of BSA-PAM-2, BSA-PAM-3 and BSA-PAM-4. (d) The Young's modulus of BSA-PAM-2, BSA-PAM-3 and BSA-PAM-4 a

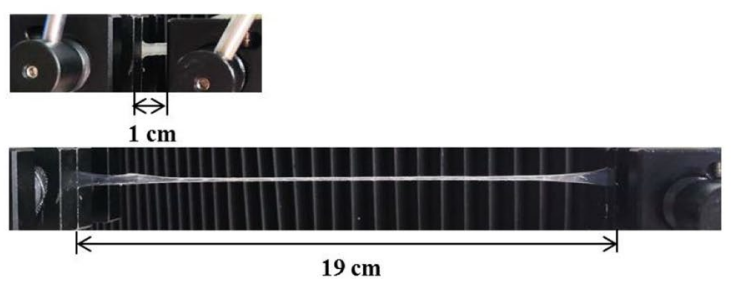

c

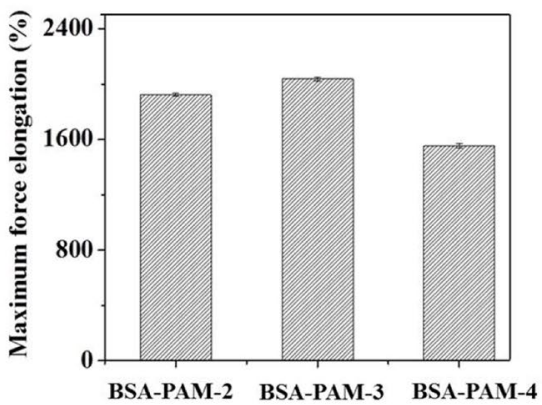

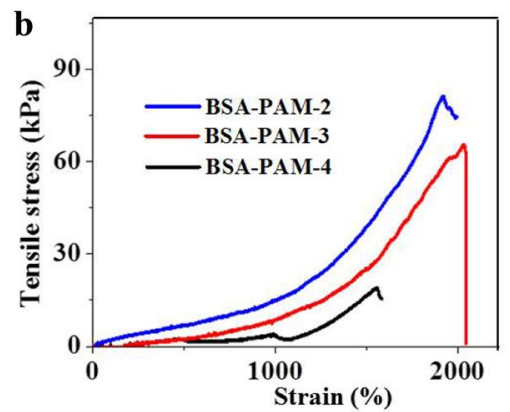

d

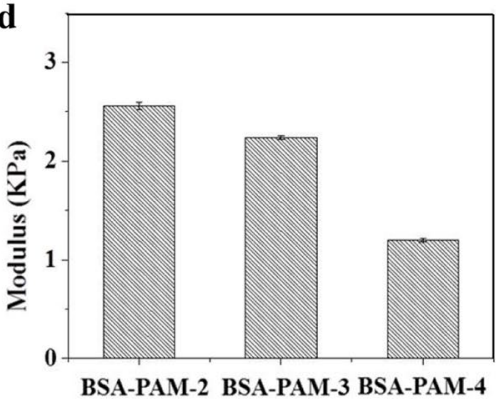

The compress tests showed that the BSA-PAM hydrogel automatically recovered its original shape after releasing the compression load (Fig. 3a), and the hysteresis loops of first cycle and second cycle almost coincided even when subjected to compress strains of up to $85 \%$, indicating the BSA-PAM hydrogel had excellent fatigue resistance caused by the dynamic nature of the hydrogen bonds between the BSA chains and PAM network (Fig. 3b). In contrast, the pure PAM hydrogel ruptured, rather than recovered, during the second tensile test [46]. With increase weight ratio of BSA : AAM, The compress modulus was gradually decreased from $216.95 \pm 6.67 \mathrm{kPa}$, $68.40 \pm 5.56 \mathrm{kPa}$ to $18.56 \pm 4.24 \mathrm{kPa}$ for BSA-PAM-2 hydrogel, BSA-PAM-3 hydrogel and BSA-PAM-4 hydrogel respectively (Table 2, Fig. 3c, d).

The super mechanical property of the BSA-PAM hydrogels can be owed to automatic breaking and recombining of hydrogen bonds between BSA chains and PAM network. In addition, the reversible association/dissociation of dynamic hydrogen bonds promoted energy dissipation when the hydrogel network was under strain testing [47].

Table 1 The parameters of tensile stress test

\begin{tabular}{llll}
\hline Samples & $\begin{array}{l}\text { Maximum force } \\
\text { elongation }(\%)\end{array}$ & $\begin{array}{l}\text { Tensile stress } \\
(\mathrm{kPa})\end{array}$ & $\begin{array}{l}\text { Yang's modulus } \\
(\mathrm{kPa})\end{array}$ \\
\hline BSA-AAM-2 & $1923 \pm 50.00$ & $81.10 \pm 6.00$ & $2.56 \pm 0.12$ \\
BSA-AAM-3 & $2037 \pm 53.20$ & $65.01 \pm 5.30$ & $2.24 \pm 0.36$ \\
BSA-AAM-4 & $1552 \pm 66.75$ & $18.96 \pm 3.40$ & $1.20 \pm 0.24$ \\
\hline
\end{tabular}

Rheology tests were used to evaluate mechanical properties of the BSA-PAM-2 hydrogel, BSA-PAM-3 hydrogel, and BSA-PAM-4 hydrogel. As shown in Fig. 4, the storage modulus $\left(\mathrm{G}^{\prime}\right)$ was obviously higher than the loss modulus $\left(\mathrm{G}^{\prime \prime}\right)$ with prolonged time, indicating that elastic component of the BSA-PAM hydrogels dominated the viscous component. In addition, $\mathrm{G}^{\prime}$ of BSA-PAM-2 was obviously higher than that of the BSA-PAM-3 hydrogel or BSA-PAM-4 hydrogel, which was measured by performing a shear frequency-sweep test using a $1 \%$ strain and an angular frequency $(\omega)$ sweeping from 0.1 to 100 $\operatorname{rad~s} s^{-1}$ (Fig. 4a). Strain sweep test $(\gamma=0.1-100 \%$ at $\omega$ $=10 \mathrm{rad} \mathrm{s}^{-1}$ ) for three hydrogels was also investigated (Fig. 4b). In the linear viscoelastic region, all hydrogels displayed typically solid-like behavior with $\mathrm{G}^{\prime}>\mathrm{G}^{\prime \prime}$. As strain increased, $\mathrm{G}^{\prime}$ and $\mathrm{G}^{\prime \prime}$ intersected, indicating that the physical crosslinking interactions in the hydrogel networks were broken. The $\gamma$ value of the intersected points of the BSA-PAM-2 hydrogel was $100 \%$ strain, which was significantly higher than BSA-PAM-3 or BSA-PAM-4 hydrogels ( $8 \%$ strain and $6 \%$ strain), which further demonstrated that the BSA-PAM-2 hydrogel had a more stable network.

\section{Adhesive property of the BSA-PAM hydrogels}

The adhesive property of BSA-PAM hydrogels was evaluated, as shown in Fig. 5. The obtained BSA-PAM hydrogel showed remarkable adhesive properties to various substrates. Taken BSA-PAM-2 hydrogel as an example 
Fig. 3 (a) The recoverability of BSA-PAM-2 hydrogel under compression. (b) loading-unloading cycles of BSA-PAM-2, BSA-PAM-3, BSA-PAM-4 at $85 \%$ strain. (c) The maximum compress stress of BSA-PAM-2, BSA-PAM-3, BSA-PAM-4. (d) The compress modulus of BSA-PAM-2, BSAPAM-3, BSA-PAM-4 a
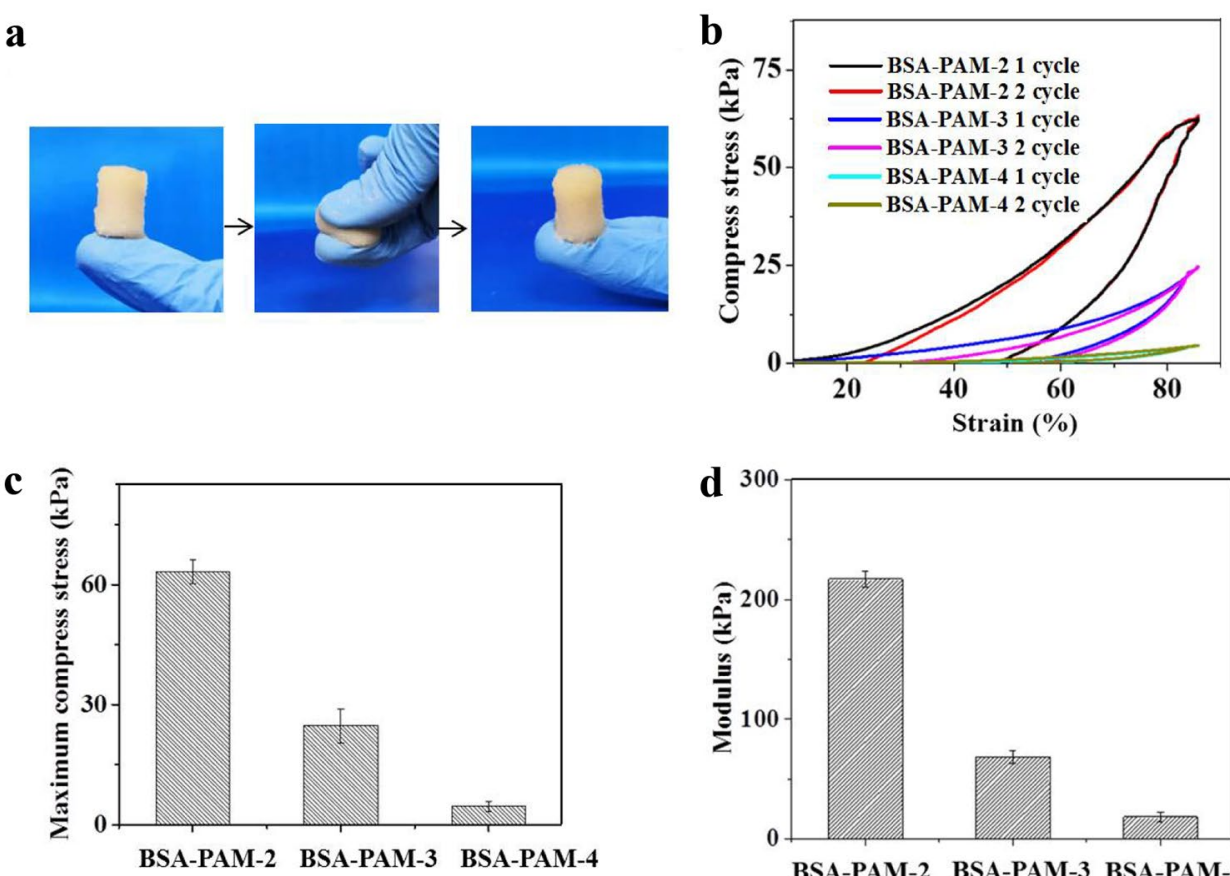

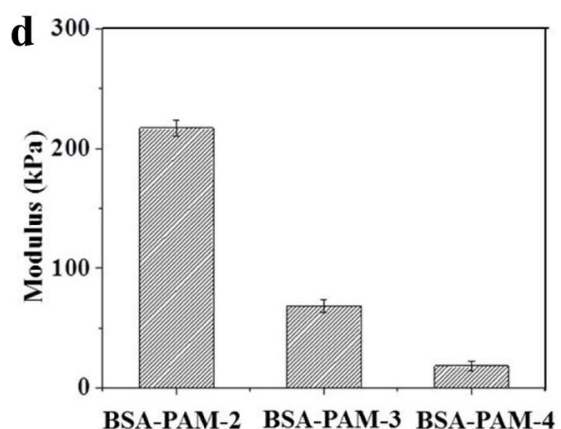

(Fig. 5a), BSA-PAM-2 hydrogel could directly adhere to various substrates including steel, ceramic, iron, glass, rubber and skin et al. Besides, the BSA-PAM hydrogel can firmly adhere a $200 \mathrm{~g}$ weight of steel. For quantitatively evaluating the adhesive strength, we used lap shear tests to systemically assess the adhesive strength of BSA-PAM-2 hydrogel, BSA-PAM-3 hydrogel and BSA-PAM-4 hydrogel to different substrates. Paper, glass, glass with gelatin and pigskin were selected as representative substrates and the results were displayed in Fig. 5b. The adhesion strength between the hydrogel and paper substrate was the highest, which was $\sim 250 \mathrm{kPa}, 150 \mathrm{kPa}$ and $120 \mathrm{kPa}$ for BSAPAM-2, BSA-PAM-3 and BSA-PAM-4 respectively due to the synergetic interactions of vander Waals interaction, hydrogen bonds et ac. Figure 5 showed that the content could significantly affect the adhesion strength of the BSAPAM hydrogel. Taking glass as an example, increasing the weight ratio of BSA : AAM from 2:1 to 4:1 would decrease the adhesion strength from $\sim 170 \mathrm{kPa}$ to $\sim 90 \mathrm{kPa}$. The decreased adhesion was likely caused by the low crosslinking degree the hydrogel network.

Table 2 The parameters of compress tests

\begin{tabular}{lcc}
\hline Samples & $\begin{array}{l}\text { Maximum compress } \\
\text { stress }(\mathrm{kPa})\end{array}$ & Compress modulus $(\mathrm{kPa})$ \\
\hline BSA-AAM-2 & $63.24 \pm 3.05$ & $216.95 \pm 6.67$ \\
BSA-AAM-3 & $24.75 \pm 4.25$ & $68.40 \pm 5.56$ \\
BSA-AAM-4 & $4.63 \pm 1.26$ & $18.56 \pm 4.24$ \\
\hline
\end{tabular}

Fig. 6 illustrated the adhesion strength between the BSA-PAM hydrogel and pigskin surfaces. The BSAPAM hydrogel were used to glue one glass gelatin slide and one fresh porcine skin, and then was photocrosslinked by UV light (Fig. 6a). The representative strain-stress curves for lap shear tests were shown in Fig. 6b, and adhesion strength was $35.09 \pm 4.01 \mathrm{kPa}$, $33.32 \pm 5.00 \mathrm{kPa}, 9.74 \pm 4.20 \mathrm{kPa}$ for BSA-PAM-2, BSA-PAM-3 and BSA-PAM-4 hydrogel respectively. For BSA-PAM-2, the adhesion strength was higher than commercial glues such as Bioglueand Fibrin (Table 3) [48-50].

The adhesive mechanism of BSA-PAM hydrogel may be owed to two factors: First, the interactions between BSA-PAM hydrogels and substrates contributed to formation of interfacial adhesion forces including vander Waals interaction, hydrogen bonding et al. [51-53]; The combination effect of physical interactions such as hydrogen bonds and covalent crosslinking created a dominant role for improving cohesion, which significantly regulating the adhesive property of the BSA-PAM hydrogels.

\section{Swelling behavior}

Swelling refers to an increase in the volume of a hydrogel after absorption of a liquid. Figure 7 demonstrated the result of the three BSA-PAM hydrogels at R. T. in water and urea. As shown, after $24 \mathrm{~h}$ of incubation, the BSA-PAM hydrogels 
Fig. 4 Rheology results of BSA-PAM-2 hydrogel, BSAPAM-3 hydrogel, BSA-PAM-4 hydrogel at (a) frequency sweep and (b) strain sweep
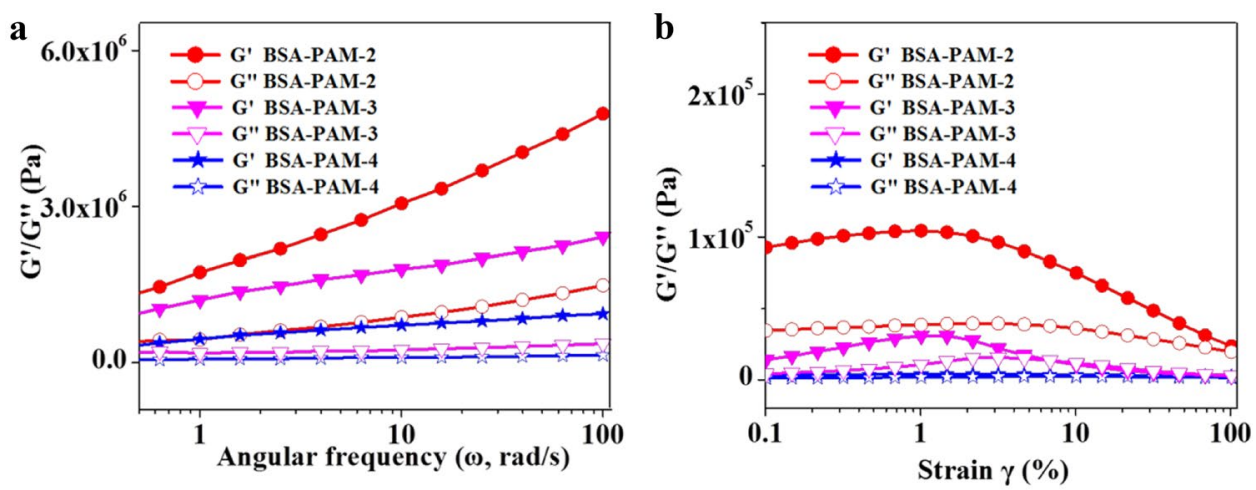

Fig. 5 (a) Adhesive performance of the BSA-PAM hydrogel. The hydrogel can adhere on surfaces of tissue (e.g. skin) (i) steel, (ii) ceramic, (iii) iron, (iv) glass, (v) rubber, (vi) steel weight of $200 \mathrm{~g}$. (b) Shear strength of BSA-PAM-2, BSA-PAM-3 and BSA-PAM-4 on different substrates
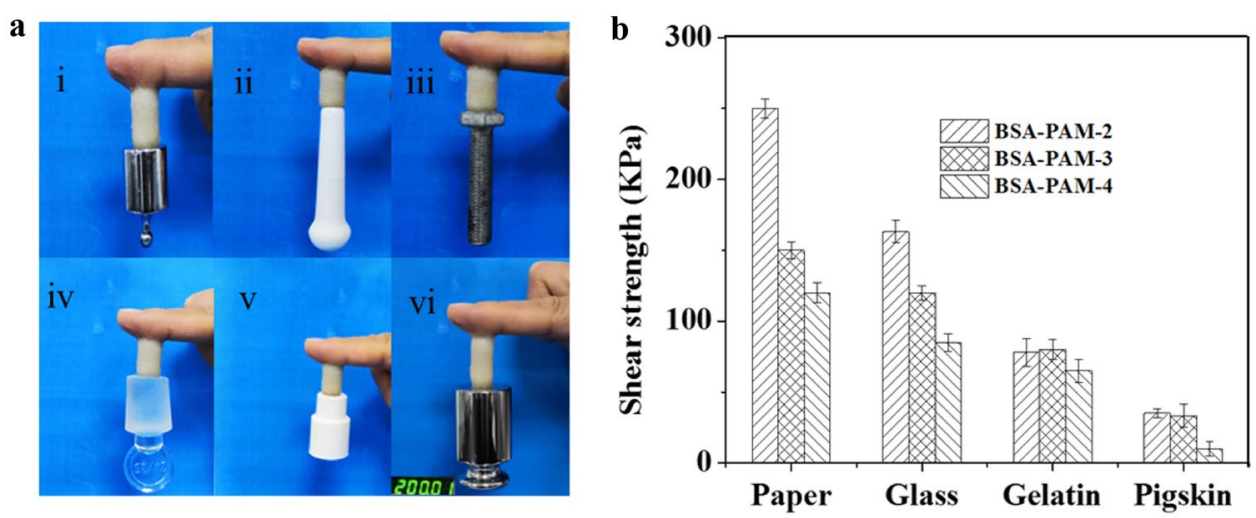

Fig. 6 (a) The modified standard test method for shear test. (b) Representative strain-stress curves for lap shear tests. (c) The bar chart of shear strength of BSA-PAM-2, BSA-PAM3and BSA-PAM-4
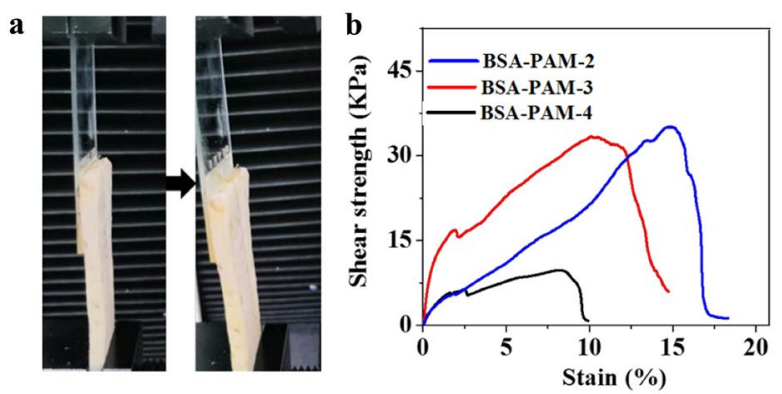

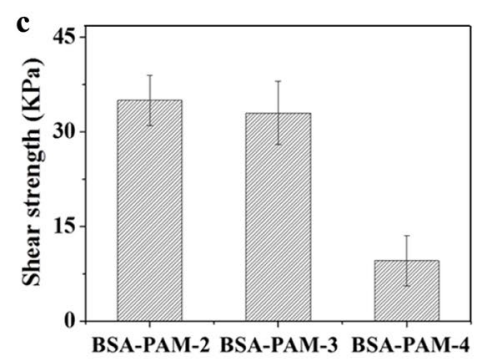

Table 3 Average shear strength data of different samples

\begin{tabular}{ll}
\hline Samples & Shear strength $(\mathrm{kPa})$ \\
\hline BSA-AAM-2 & $35.09 \pm 4.0$ \\
BSA-AAM-3 & $33.32 \pm 5.0$ \\
BSA-AAM-4 & $9.74 \pm 4.2$ \\
BioGlue [48] & $20.0-30.0$ \\
Fibrin gule [49, 50] & $0.7-27.0$ \\
\hline
\end{tabular}

exhibited varying degrees of swelling in water and urea. After $48 \mathrm{~h}$ incubation, swelling of the BSA-PAM hydrogels achieved equilibrium. It was clear that the swelling ratio of the BSA-PAM hydrogels in urea was obviously higher than those in water, due to the intermolecular hydrogen bonds had been destroyed, indicating that the hydrogen bonds were an important driving force for formation of BSA-PAM hydrogel (Fig. 7a-c). 

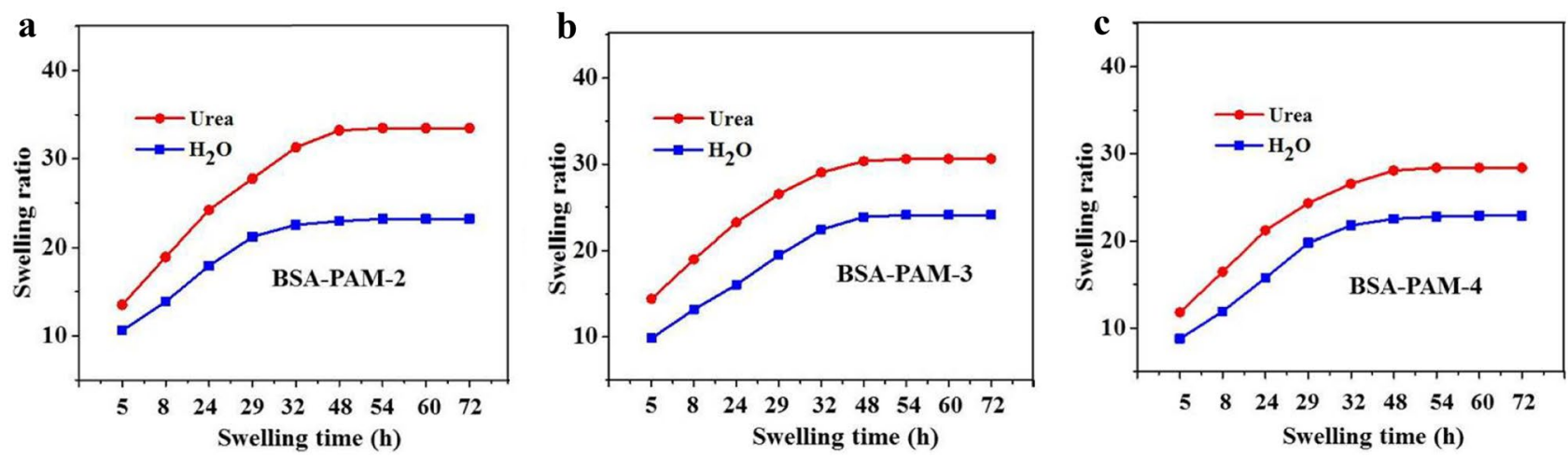

Fig. 7 Swelling kinetics results of (a) BSA-PAM-2 hydrogel, (b) BSA-PAM-3 hydrogel, (c) BSA-PAM-4 hydrogel in urea and water

\section{Conclusions}

This study presented BSA-PAM hydrogels which were constructed through combination of physical interactions including hydrogen bonding and chain entanglements, and chemical covalent photo-crosslinking. The BSA chains interdiffused and got physically entangled within the PAM network. Results showed that BSA-PAM hydrogels displayed excellent mechanical and adhesive properties, and maximum force elongation reached $1552 \%$ 2037\%, and the adhesiveness was $90 \mathrm{kPa} \sim 150 \mathrm{kPa}$ for glass and $9.74 \mathrm{kPa} 35.09 \mathrm{kPa}$ for pigskin respectively. This work provided a facile way of fabricating high mechanical and adhesive hydrogels.

Supplementary information The online version contains supplementary material available at https://doi.org/10.1007/s10965-021-02765-x.

Acknowledgments This research was supported by special fund of Guangdong academy of sciences (No. 2020GDASYL-20200103042, No. 2019GDASYL-0103020, 2019GDASYL-0402002 and 2019GDASYL-0105009), National Natural Science Foundation of China (No. 21907037).

Open Access This article is licensed under a Creative Commons Attribution 4.0 International License, which permits use, sharing, adaptation, distribution and reproduction in any medium or format, as long as you give appropriate credit to the original author(s) and the source, provide a link to the Creative Commons licence, and indicate if changes were made. The images or other third party material in this article are included in the article's Creative Commons licence, unless indicated otherwise in a credit line to the material. If material is not included in the article's Creative Commons licence and your intended use is not permitted by statutory regulation or exceeds the permitted use, you will need to obtain permission directly from the copyright holder. To view a copy of this licence, visit http://creativecommons.org/licenses/by/4.0/.

\section{References}

1. Bastings MMC, Koudstaal S, Kieltyka RE, Nakano Y, Pape ACH, Feyen DAM, van Slochteren FJ, Doevendans PA, Sluijter JPG, Meijer EW, Chamuleau SAJ, Dankers PYW (2014) A fast pH-switchable and self-healing supramolecular hydrogel carrier for guided, local catheter injection in the infarcted myocardium. Adv Healthc Mater 3:70-78

2. Wang WD, Narain R, Zeng H (2018) Rational design of selfhealing tough hydrogels: A mini review. Front Chem 6:497

3. Wichterle O, Lim D (1960) Hydrophilic gels for biological use. Nature 185:117-118

4. Cai ZW, Saiding Q, Cheng L, Zhang LC, Wang Z, Wang F, Chen XL, Chen G, Deng LF, Cui WG (2021) Capturing dynamic biological signals via bio-mimicking hydrogel for precise remodeling of soft tissue. Bioact Mater 6:4506-4516

5. Yang Z, Yang XW, Long R, Li JY (2021) Stimulation modulates adhesion and mechanics of hydrogel adhesives. Langmuir 37:7097-7106

6. Yang Z, Ma ZW, Liu SY, Li JY (2021) Tissue adhesion with tough hydrogels: Experiments and modeling. Mech Mater 157:103800

7. Yang B, Song JL, Jiang YH, Li M, Wei JJ, Qin JJ, Peng WJ, Lasaosa FL, He YY, Mao HL, Yang J, Gu ZW (2020) Injectable adhesive self-healing multicross-linked double-network hydrogel facilitates full-thickness skin wound healing. ACS Appl Mater Inter 12:57782-57797

8. Sharifi S, Islam MM, Sharifi $\mathrm{H}$ et al (2021) Tuning gelatin-based hydrogel towards bioadhesive ocular tissue engineering applications. Bioact Mater 6:3947-3961

9. Qiao ZW, Lv XL, He SH et al (2021) A mussel-inspired supramolecular hydrogel with robust tissue anchor for rapid hemostasis of arterial and visceral bleedings. Bioact Mater 6:2829-2840

10. Han K, Bai Q, Wu WD, Sun N, Cui N, Lu TL (2021) Gelatinbased adhesive hydrogel with self-healing, hemostasis, and electrical conductivity. Int J Biol Macromol 183:2142-2151

11. Han W, Zhou B, Yang K et al (2020) Biofilm-inspired adhesive and antibacterial hydrogel with tough tissue integration performance for sealing hemostasis and wound healing. Bioact Mater 5:768-778

12. Preman NK, Priya SES, Prabhu A, Shaikh SB, Vipin C, Barki RR, Bhandary YP, Rekha PD, Johnson RP (2020) Bioresponsive supramolecular hydrogels for hemostasis, infection control and accelerated dermal wound healing. J Mater Chem B 8:8585-8598

13. Costa RR, da Costa DS, Reis RL, Pashkuleva I (2019) Bioinspired baroplastic glycosaminoglycan sealants for soft tissues. Acta Biomater 87:108-117

14. Kazemzadeh-Narbat M, Annabi N, Khademhosseini A (2015) Surgical sealants and high strength adhesives. Mater Today 18:176-177

15. Bal-Ozturka A, Cecen B, Avci-Adali M, Topkaya SN, Alarcin E, Yasayan G, Li Y CE, Bulkurcuoglu B, Akpek A, Avci H, Shi K, Shin SR, Hassan S (2021) Tissue adhesives: from research to clinical translation. Nano Today 36:101049 
16. Mehdizadeh M, Yang J (2013) Design strategies and applications of tissue bioadhesives. Macromol Biosci 13:271-288

17. Yang JW, Bai RB, Chen BH, Suo ZG (2019) Hydrogel adhesion: a supramolecular synergy of chemistry, topology, and mechanics. Adv Funct Mater 30:1901693

18. Cerda DG, Ballester AM, Aliena-Valero A, Caraben-Redano A, Lloris JM (2015) Use of cyanoacrylate adhesives in general surgery. Surg Today 45:939-956

19. Spotnitz WD, Burks S (2012) Hemostats, sealants, and adhesives III: a new update as well as cost and regulatory considerations for components of the surgical toolbox. Transfusion 52:2243-2255

20. Bouten PJM, Zonjeea M, Bender J, Yauw STK, van Goor H, van Hest JCMV, Hoogenboom R (2014) The chemistry of tissue adhesive materials. Prog Polym Sci 39:1375-1405

21. Annabi N, Zhang YN, Assmann A, Sani ES, Cheng G, Lassaletta AD, Vegh A, Dehghani B, Ruiz-Esparza GU, Wang XC, Gangadharan S, Weiss AS, Khademhosseini A (2017) Engineering a highly elastic human protein-based sealant for surgical applications. Sci Transl Med 9:eaai7466

22. Tsai YL, Theato P, Huang C F, Hsu SH (2020) A 3D-printable, glucosesensitive and thermoresponsive hydrogel as sacrificial materials for constructs with vascular-like channels. Appl Mater Today 20:100778

23. Wu CH, Tu CW, Aimi J, Zhang JW, Chen T, Wang CC, Huang CF (2020) Mechanochromic double network hydrogels as a compression stress sensor. Polym Chem 11:6423-6428

24. Cheng KC, Huang CF, Wei Y, Hsu SH (2019) Novel chitosancellulose nanofiber selfhealing hydrogels to correlate self-healing properties of hydrogels with neural regeneration effects. NPG Asia Mater 11:25

25. Hirsch M, Charlet A, Amstad E (2020) 3D printing of strong and tough double network granular hydrogels. Adv Funct Mater 31:2005929

26. Gong JP, Katsuyama Y, Kurokawa T, Osada Y (2003) Doublenetwork hydrogels with extremely high mechanical strength. Adv Mater 15:1155-1158

27. Tu CW, Tsai FC, Chen JK, Wang HP, Lee RH, Zhang JW, Chen T, Wang CC, Huang CF (2020) Preparations of tough and conductive PAMPS/PAA double network hydrogels containing cellulose nanofibers and polypyrroles. Polymers 12:2835

28. Chen J, Ma XY, Dong QC, Song DH, Hargrove D, Vora SR, Ma AWK, Lu XL, Lei Y (2016) Self-healing of thermally-induced, biocompatible and biodegradable protein hydrogel. RSC Adv 6:56183-56192

29. Chen J, Dong QC, Ma X, Fan TH, Lei Y (2016) Repetitive biomimetic self-healing of $\mathrm{Ca}^{2+}$-induced nanocomposite protein hydrogels. Sci Rep 6:30804

30. Bahouth Z, Moskovitz B, Halachmi S, Nativ O (2017) Bovine serum albumin-glutaraldehyde (BioGlue ${ }^{\circledR}$ ) tissue adhesive versus standard renorrhaphy following renal mass enucleation: a retrospective comparison. Ther Adv Urol 9:67-72

31. Hidas G, Kastin A, Mullerad M, Shental J, Moskovitz B, Nativ O (2006) Sutureless nephron-sparing surgery: use of albumin glutaraldehyde tissue adhesive (BioGlue). Urology 67:697-700

32. Zhu WZ, Peck Y, Iqbal J, Wang DA (2017) A novel DOPAalbumin based tissue adhesive for internal medical applications. Biomaterials 147:99-115

33. Rusu AG, Chiriac AP, Nita LE, Mititelu-Tartau L, Tudorachi N, Ghilan A, Rusu D (2019) Multifunctional BSA scaffolds prepared with a novel combination of UV-crosslinking systems. Macromol Chem Phys 220:1900378

34. Sun YL, Huang YB (2016) Disulfide-crosslinked albumin hydrogels. J Mater Chem B 4:2768-2775

35. Upadhyay A, Kandi R, Rao CP (2018) Injectable, self-healing, and stress sustainable hydrogel of BSA as a functional biocompatible material for controlled drug delivery in cancer cells. ACS Sustain Chem Eng 6:3321-3330
36. Liu Z, Tang ZQ, Zhu L, Lu SP, Chen F, Tang C, Sun H, Yang J, Qin G, Chen Q (2019) Natural protein-based hydrogels with high strength and rapid self-recovery. Int J Biol Macromol 141:108-116

37. Tang ZQ, Chen Q, Chen F, Zhu L, Lu SP, Ren BP, Zhang YX, Yang J, Zheng J (2019) General principle for fabricating natural globular protein-based double-network hydrogels with integrated highly mechanical properties and surface adhesion on solid surfaces. Chem Mater 31:179-189

38. Rodrigues SB, Collares FM, Leitune VCB, Schneider LFJ, Ogliari FA, Petzhold CL, Samuel SMW (2015) Influence of hydroxyethyl acrylamide addition to dental adhesive resin. Dent Mater 31:1579-1586

39. Fugolin APP, Navarro O, Logan MG, Huynh V, Franca CM, Ferracane JL, Pfeifer CS (2020) Synthesis of di- and triacrylamides with tertiary amine cores and their evaluation as monomers in dental adhesive interfaces. Acta Biomater 115:148-159

40. Li J, Celiz AD, Yang J, Yang Q, Wamala I, Whyte W, Seo BR, Vasilyev NV, Vlassak JJ, Suo Z, Mooney DJ (2017) Tough adhesives for diverse wet surfaces. Science 357:378-381

41. Sun JY, Zhao XH, Illeperuma WRK, Chaudhuri O, Oh KH, Mooney DJ, Vlassak JJ, Suo ZG (2012) Highly stretchable and tough hydrogels. Nature 489:133-136

42. Lang N, Pereira MJ, Lee Y et al (2014) A blood-resistant surgical glue for minimally invasive repair of vessels and heart defects. Sci Transl Med 6:218ra6

43. Adewunmi AA, Ismail S, Sultan AS, Ahmad Z (2017) Performance of fly ash based polymer gels for water reduction in enhanced oil recovery: gelation kinetics and dynamic rheological studies. Korean J Chem Eng 34:1638-1650

44. Yi Q, Li C, Manlai Z, Yuli L, Ruiquan L (2017) Dynamic thickening investigation of the gelation process of PAM/PEI system at high temperature and high pressure. J Disper Sci Technol 38:1640-1646

45. Adewunmi AA, Ismail S, Sultan AS (2015) Laboratory scale study on rheological behavior, morphological and structural properties of crosslinked polyacrylamide composite hydrogels embedded with date seed powder. J Appl Polym Sci 132:42110

46. Han L, Yan LW, Wang KF et al (2017) Tough, self-healable and tissue-adhesive hydrogel with tunable multifunctionality. NPG Asia Mater 9:e372

47. Chen JS, Liu JF, Thundat T, Zeng HB (2019) Polypyrrole-doped conductive supramolecular elastomer with stretchability, rapid self-healing, and adhesive property for flexible electronic sensors. ACS Appl Mater Interfaces 11:18720-18729

48. Sugiura Y, Okada M, Hirano K, Matsumoto T (2021) Bone mineral analogue ceramic block as an instant adhesive to biological soft tissue. Adv Mater Interfaces 8:2002032

49. Sierra DH, Feldman DS, Saltz R, Huang S (1992) A method to determine shear adhesive strength of fibrin sealants. J Appl Biomater 3:147-151

50. Chao HH, Torchiana DF (2003) BioGlue: albumin/glutaraldehyde sealant in cardiac surgery. J Card Surg 18:500-503

51. Xiao WQ, Qu XH, Li JL, Chen L, Tan YF, Li KJ, Li B, Li XL (2019) Synthesis and characterization of cell-laden double-network hydrogels based on silk fibroin and methacrylated hyaluronic acid. Eur Polym J 118:382-392

52. Matsuda M, Ueno M, Endo Y, Inoue M, Sasaki M, Taguchi T (2019) Enhanced tissue penetration-induced high bonding strength of a novel tissue adhesive composed of cholesteryl group-modified gelatin and disuccinimidyl tartarate. Colloids Surf B 91:48-56

53. Sun J, Su JJ, Ma C, Gostl R, Herrmann A, Liu K, Zhang HJ (2020) Fabrication and mechanical properties of engineered proteinbased adhesives and fibers. Adv Mater 32:1906360

Publisher's Note Springer Nature remains neutral with regard to jurisdictional claims in published maps and institutional affiliations. 